\title{
Adenocarcinomas mínimos de próstata en la biopsia sometidos a prostatectomía radical
}

\author{
M. Montesino Semper, J. Jiménez Aristu, P. Fernández Seara*, C. Sarmiento Gómez,
} L. Ripa Saldías, A. Rivas Alonso, M. Pinós Paul, Á. de Pablo Cárdenas, I. Villanueva Pérez,

A. Santiago González de Garibay.

Servicio de Urología y *Anatomía-Patológica. Hospital Virgen del Camino. Pamplona.

Actas Urol Esp 2005; 29 (5): 481-484

\section{RESUMEN}

ADENOCARCINOMAS MÍNIMOS DE PRÓSTATA EN LA BIOPSIA SOMETIDOS A PROSTATECTOMÍA RADICAL

Fundamento: Valoración de datos clínico-patológicos de pacientes sometidos a prostatectomía radical por mínimo adenocarcinoma prostático en la biopsia.

Métodos: Análisis retrospectivo de pacientes intervenidos de prostatectomía radical por mínimo adenocarcinoma, frente al resto de prostatectomías radicales.

Resultados: En 20 pacientes (7,6 \%), de los 260 sometidos a prostatectomía radical entre 1992 y 2004 , se definió la biopsia como "mínimo adenocarcinoma". Tenían edades entre 58 y 73 años y los PSA entre 5,2 y 17,1 ng/ml. Todos, excepto uno eran clínicamente T1c. En la anatomía-patológica definitiva el Gleason fue de 6, 4, 3 y 2, en 3, 3, 8 y 4 pacientes respectivamente, con uno con mínimo adenocarcinoma no graduado y sólo un PIN-III en otro. Tres presentaron un solo foco con un volumen tumoral inferior al 5\% del tejido (el 84,2 \% con tumor significativo). El estadio final fue 1 pTO (PIN III), 7 pT2a, 11 pT2b y 1 pT3a $(62,5 \%$ bilaterales).

Con respecto al resto de pacientes prostatectomizados, los pacientes con mínimo adenocarcinoma presentaron diferencias significativas en los Gleason $(p=0,029)$ y los estadios $(p=0,02)$; no en la media del PSA ( $p=0,243)$.

CONCLUSIONES: Los adenocarcinomas de próstata mínimos en la biopsia son significativos, aunque presentan estadios y grados inferiores al resto.

Palabras clave: Adenocarcinoma de próstata. Anatomía patológica. Próstata.

\section{ABSTRACT}

MINIMAL PROSTATIC ADENOCARCINOMAS IN THE BIOPSY TREATED WITH RADICAL PROSTATECTOMY

Fundamentals: Valuation about clinical pathologyc facts of patients having undergone a radical prostatectomy due to a minimal prostate adenocarcinoma shown at the biopsy.

Methods: Retrospective analysis of patients having undergone a radical prostatectomy due to a minimal prostate adenocarcinoma shown at the biopsy in front of the remaining radical prostatectomies.

Results: In 20 patients $(7,6 \%)$ out of the 260 having undergone a radical prostatectomy between 1992 and 2004 the biopsy was informed as "minimal adenocarcinoma". These patients ranged 58 to 73 years with PSA levels from 5.2 to $17.1 \mathrm{ng} . / \mathrm{ml}$. Everyone except one were clinically T1c. At the definitive pathological study the Gleason was 6, 4, 3 and 2 in 3, 3, 8 and 4 patients respectively, with one having a minimal adenocarcinoma not graded and another one with a PIN ?. 3 showed only 1 focus with a tumoral volume less than $5 \%$ of the tissue (84.2\% with significant tumor or multifocal). The final staging was 1 pTO (PIN ?), 7 pT2a, 11 pT2b and 1 pT3a (62.5\% bilaterals).

Relating to the remaining patients under prostatectomy, patients with minimal adenocarcinoma presented significative differences in Gleason sum ( $<$ 0.029) and staging ( $p=0.02)$; no in PSA mean $(p=0,243)$.

Summary: Minimal adenocarcinomas of the prostate at the biopsy are significant but do present lower staging and grading in relation with the rest of patients.

Keywords: Adenocarcinoma of the prostate. Pathological study. Prostate. 
$\mathrm{E}$ n los últimos años, el creciente aumento en las determinaciones del PSA, ha conducido igualmente a un incremento en el número de biopsias, así como al diagnóstico de tumores prostáticos de menor tamaño, con un aumento del riesgo de confusión con condiciones benignas ${ }^{1}$. Nos encontramos cada vez más a menudo con informes de nuestros patólogos indicándonos la presencia de "mínimos focos de adenocarcinoma de próstata" en las biopsias. Algunos de estos pacientes podrían ser sometidos a tratamiento radical innecesario por presentar tumores clínicamente insignificantes.

Pretendemos valorar los aspectos clínico-patológicos de nuestros pacientes que, con el diagnóstico de "mínimo foco de adenocarcinoma" en la biopsia, han sido sometidos a prostatectomía radical.

\section{PACIENTES Y METODOS}

Realizamos un estudio retrospectivo de nuestra base de datos de los pacientes sometidos a prostatectomía radical por carcinoma de próstata entre enero de 1992 y de enero de 2004. Las biopsias de próstata, realizadas en sextante con aguja 18G y dirigidas ecográficamente por vía transrectal, fueron interpretadas como de "mínimo adenocarcinoma" cuando existía un foco en uno o dos de los 6 cilindros de la muestra y con un número de glándulas afectadas de cinco o menor en cada foco. A ninguno de estos tumores se le asignó Gleason en la biopsia.

Se recogen la edad de los pacientes al tratamiento, el PSA al diagnóstico, el estadio clínico, el grado histológico de la pieza aplicando la suma de Gleason (sumando los dos patrones predominantes), el volumen tumoral en porcentaje y el estadio patológico definitivo según la clasificación TNM de la UICC de 1997.

Los cortes de las piezas se hicieron cada $3 \mathrm{~mm}$ y ocasionalmente se realizaron técnicas de inmunohistoquímica con queratina de alto peso molecular (34BE12) y/o p63. El volumen tumoral se entendió significativo si era igual o superior al 5\% o existía afectación bilateral o multifocal. Se realizó el análisis estadístico con el programa informático SPSS -11.5, comparando la media del PSA, las sumas de Gleason bajas o moderadas (entre 2 y 6) y los estadios anatomopatológicos órganoconfinados (igual o inferior a pT2b), de este grupo de pacientes frente al resto de los sometidos a prostatectomía radical. Para las medias de PSA se empleó la $t$ de Student si la variable seguía una distribución normal, y la U de Mann- Whitney en caso contrario. Para los grados y estadios, el Ji al cuadrado $\left(\chi^{2}\right)$; si alguno de los valores esperados era menor de 5, empleamos el test exacto de Fisher. Se aceptó como nivel de significación estadística el del 5\%.

\section{RESULTADOS}

En 20 pacientes $(7,6 \%)$, de los 260 sometidos a prostatectomía radical entre 1992 y 2004, se definió la biopsia como "mínimo adenocarcinoma de próstata"; todos con biopsia en sextante. Estos tenían edades entre 58 y 73 años y un PSA al diagnóstico entre 5,2 y $17,1 \mathrm{ng} / \mathrm{ml}$, con una media de $8,4 \mathrm{ng} / \mathrm{ml}$ y una mediana de $7,4 \mathrm{ng} / \mathrm{ml}$. Ninguno de ellos llevó tratamiento hormonal neoadyuvante. Todos, excepto uno, estadiado como T2a, presentaban una próstata normal al tacto rectal y por tanto eran clínicamente T1c. En dos pacientes el diagnóstico se realizó en una segunda biopsia y en uno en una cuarta.

En la anatomía-patológica definitiva el Gleason fue de $6,4,3$ y 2, en 3, 3, 8 y 4 pacientes respectivamente (Fig. 1). En uno sólo se encontró un mínimo adenocarcinoma que no fue graduado y en otro sólo se detectó un PIN III. De los 19 pacientes con tumor, 3 presentaron un único foco con un volumen tumoral inferior al $5 \%$

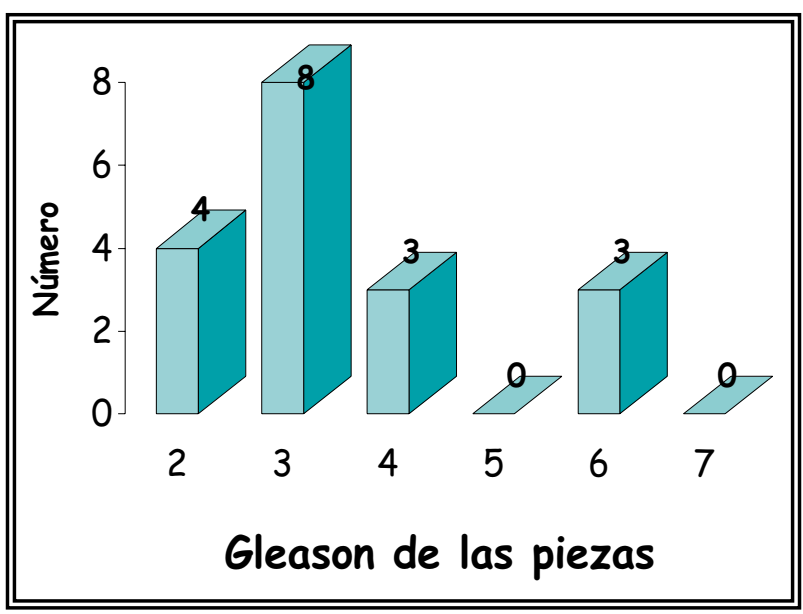

FIGURA 1. Distribución de las sumas de Gleason en las piezas de prostatectomia radical de los carcinomas minimos en la biopsia. 
del tejido. El estadio anatomopatológico final fue: 1 pTO (PIN III), 7 pT2a, 11 pT2b y 1 pT3a, con bordes positivos en dos, ambos laterales (Fig. 2).

Al hacer la comparación con el resto de pacientes sometidos a prostatectomía radical, los pacientes con mínimo adenocarcinoma presentaban diferencias significativas en:

las sumas de Gleason, todas en grados bajos o moderados, frente al 79,7\% en el resto, con una $\mathrm{p}=0,029 ; \mathrm{y}$

el estadio anatomopatológico, órganoconfinado en todos salvo en $1(5,2 \%)$, que presentó un estadio pT3a. El 29,1 \% del resto de pacientes tenían un estadio pT3a o superior $(p=0,020)$.

$\mathrm{El}$ análisis estadístico de la media del PSA $(8,4$ $\mathrm{ng} / \mathrm{ml}$ ) de estos pacientes no mostró diferencias significativas frente al resto de los pacientes prostatectomizados (media de PSA: 11,2 ng/ml).

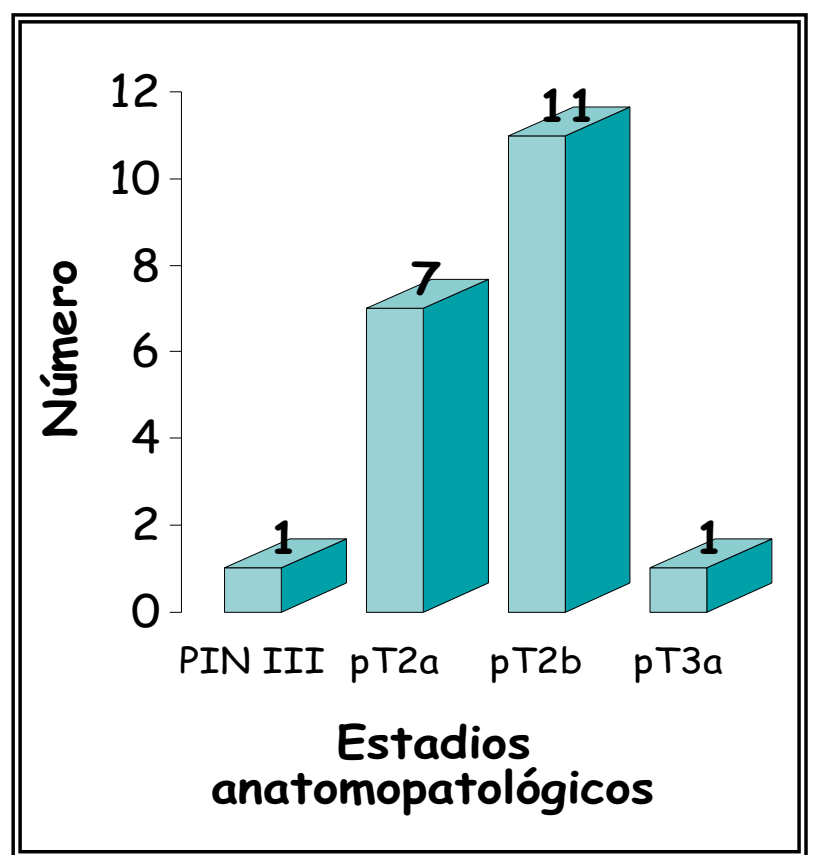

FIGURA 2: Estadios anatomopatológicos de los carcinomas de próstata minimos en la biopsia.

\section{DISCUSIÓN}

La cantidad de tumor prostático encontrado en las biopsias es hoy en día un factor pronóstico reconocido $^{1,2}$, y predictivo de enfermedad órganoconfinada ${ }^{3}$. Sin embargo, la definición de lo que es carcinoma mínimo en la biopsia varía según diferentes autores. Así Allan et al. en su revisión de carcinomas mínimos, lo definen como la presencia de un único foco de $0,5 \mathrm{~mm}$ en un solo cilindro de las biopsias, para una media de 6,3 cilindros por biopsia y con un Gleason igual o menor de $6^{4}$. Otros autores como Rubin o Leroy establecen como punto de corte la afectación de menos de $1 \mathrm{~mm}$ o < del 5\% de la biopsia sin atender al Gleason ${ }^{5,6}$.

Por otra parte, el diagnóstico patológico de mínimo carcinoma en las biopsias se está dando con una frecuencia creciente en los últimos años. Esto hace que en función de los años revisados y criterios empleados, los porcentajes de incidencia de estos mínimos carcinomas varien entre un 10,7\% de Rubin ${ }^{5}$ y un $2,7 \%$ de Allan $^{4}$, con porcentajes similares a los nuestros de D'Amico ${ }^{7}$ o Leroy ${ }^{6}$. En nuestros pacientes el patólogo no asignó el Gleason en las biopsias. La escasa correlación entre el Gleason de estas biopsias con mínimos carcinomas y el de las piezas, y por tanto su poca capacidad predictiva, llevó a Rubin a afirmar que pueda no ser apropiado darlo en estos pacientes ${ }^{5}$.

Los resultados en las piezas de prostatectomía son igualmente variables, aunque de una manera general los diferentes autores llegan a una misma conclusión: estos tumores son en su mayoría patológicamente significativos. Los criterios para determinar que un carcinoma de próstata no es significativo en la pieza se basan en un tamaño tumoral inferior a $0,5 \mathrm{cc}$, márgenes negativos y ningún Gleason 4 ó 5 o sumas de Gleason $=o ́$ < a 6 6,8. Sin embargo Thorson et al. ${ }^{9}$ establecen el límite en 0,2 cc y tienen en cuenta el volumen total de los posibles microtumores frente a otros autores que sólo consideran el del predominante ${ }^{4}$. En nuestros casos se evaluó el volumen tumoral en la pieza de prostatectomía en porcentaje. No existe un consenso a la hora de emplear un método que mida el volumen tumoral ${ }^{10} \mathrm{y}$ aunque los métodos estereológicos para determinarlo puedan ser los más exactos, suponen un trabajo y un consumo de tiempo difícilmente asumible por la mayoría, por lo que se ha recomendado desde la Sociedad Europea de Uropatología, entre otras, emplear el porcentaje de tejido afectado por el tumor ${ }^{11}$.

Teniendo en cuenta lo expuesto, D`Amico et al. encuentran en su serie de 66 pacientes, una afectación de al menos el 50\% de un lóbulo en el $92 \%$ de ellos, estando el $94 \%$ de los tumores órganoconfinados, el 89\% con márgenes negati- 
vos, en el $79 \%$ un Gleason $=0<$ de 6 y con $10 \%$ de recidivas bioquímicas a los 5 años ${ }^{7}$. Por su parte Thorson et al. en 50 pacientes encuentran un $82 \%$ de carcinomas patológicamente significativos $^{9}$. Igualmente Leroy en 24 tumores aprecia 7 con afectación bilateral y 2 con extensión extracapsular, y todos excepto 3 con Gleason en la pieza igual ó inferior a $6^{6}$. Sin embargo Allan et al. en sus 54 pacientes establecen que el $67 \%$ son clínicamente insignificantes: sus criterios de inclusión son más restrictivos (menos de $0,5 \mathrm{~mm}$ en la biopsia ) y para el volumen tumoral sólo tiene en cuenta el predominante ${ }^{4}$.

En nuestros pacientes consideramos que la afectación bilateral (12), o la unilateral múltiple (1) o única superior al $5 \%$ del lóbulo eran criterios suficientes para considerar el adenocarcinoma de próstata patológicamente significativo. La presencia de sumas de Gleason en la pieza, en todos los casos inferiores a 7 , guarda relación con los datos publicados previamente de toda nuestra serie, donde sólo el $12 \%$ de las piezas presentaban Gleason igual o superior a $7^{12}$.

Tanto Epstein et al., en pacientes con tumores T1c, como Allan et al., en estos tumores mínimos, han buscado en los datos preoperatorios fórmulas que permitan establecer a priori qué tumores resultarán insignificantes. Los primeros, mediante la relación del PSA libre/ total con un punto de corte de 0,15 , junto a los datos de la biopsia (menos de 3 cilindros y menos del 50\% cada uno, Gleason de 6 o menor y ningún 4 ó 5), obtenían un valor predictivo positivo del $94,4 \%$ pero un negativo del $77,2 \%$, detectando de esta manera sólo el $34 \%$ de los definidos insignificantes. Los segundos, con la densidad del PSA de 0,15 o menor obtienen un $83,3 \%$ y un $66,7 \%$ de valores predictivos positivos y negativos respectivamente. Quizá estos datos puedan tenerse en cuenta en ciertos pacientes, junto con otros factores como comorbilidad o edad, para plantearnos otras alternativas terapéuticas no agresivas $^{4,8}$. O bien disponernos para una actitud de expectación armada como la recientemente propuesta por Mohamad y Ballentine ${ }^{13}$.

En conclusión y en base a nuestros datos, los adenocarcinomas mínimos de próstata en la biopsia, presentan en su mayoría volúmenes significativos, pero en unos estadios y grados inferiores al resto de pacientes con adenocarcinoma, y de manera estadísticamente significativa.

\section{Agradecimientos}

A las Dras. Begoña Bermejo Fraile y Nerea Egués Olazábal por su inestimable ayuda en el análisis estadístico de los datos.

\section{REFERENCIAS}

1. Algaba F, Epstein JI, Aldape $\mathrm{HC}$ at al. Assessment of prostate carcinoma in core needle biopsy. Definition of minimal criteria for the diagnosis of cancer in biopsy material. Cancer 1996;78:376-381.

2. Freedland SJ, Csathy GS, Dorey F, Aronson WJ. Percent prostate tissue with cancer is more predictive of biochemical failure or adverse pathology after radical prostatectomy than prostate specific antigen or Gleason score. J Urol 2002;167:516-520.

3. Villamon Fort R, Martínez Jabaloyas JM, Gil Salom M et al. El porcentaje de tumor en cilindros de biopsia prostática como factor pronóstico de enfermedad órganoconfinada en pacientes candidatos a prostatectomía radical. Actas Urol Esp 2001;25:493-498.

4. Allan R W, Sanderson H, Epstein J I. Correlation of minute ( $0,5 \mathrm{~mm}$ or less) focus of prostate adenocarcinoma on needle biopsy with radical prostatetctomy specimen: role of prostate specific antigen density. J Urol 2003;170:370-372.

5. Rubin MA, Dunn R, Kambham N et al. Should a Gleason score be assigned to a minute focus of carcinoma on prostate biopsy?. Am J Surg Pathol 2000;24:1634-1640.

6. Leroy X, Aubert, Villers A et al. Minimal fous of adenocarcinoma on prostate biosy: clinicopathological correlations. J Clin Pathol 2003;56:230-232.

7. D'Amico A V, Wu Y, Chen M H et al. Pathologic findings and prostate specific antigen oucome after radical prostatectomy for patients diagnosed on the basis of a single microscopic focus of prostate carcinoma with Gleason score $\leq 7$. Cancer, 2000;89:1810-1817.

8. Epstein JI, Chan D W, Sokol LJ, et al. Nonpalpable stage T1c prostate cancer: prediction of insignificant disease using free/total prostate specific antigen levels and needle biopsy findings. J Urol 1998;160:2407-2411.

9. Thorson P, Vollmer RT, Arcangeli C, et al. Minimal carcinoma en prostate needle biopsy specimens: diagnostic features and radical prostatectomy follow-up. Mod Pathol 1998;11:543-551.

10. Bostwick DG, Grignon DJ, Hammond ME, et al. Pronostic factors in prostate cancer. College of American Pathologists Consensus Statement 1999. Arch Pathol Lab Med 2000; 124:995-1000.

11. Montironi R, van der Kwast Th, Boccon-Gibod L, Bono A, Boccon-Gibod L. Handling and pathology reporting of radical prostatectomy specimens. Eur Urol 2003;44:626-636.

12. Montesino Semper M, Jiménez Aristu J, Repáraz Romero B, et al. Estudio de correlación entre las sumas de Gleason de las biopsias de próstata con adenocarcinoma y de las piezas de prostatectomía radical. Arch Esp Urol 2004;57: 519-523.

13. Mohamad A, Ballentine C $\mathrm{H}$. Update on watchful waiting for prostate cancer. Curr Opin Urol 2004;14:171-175.

Dr. M. Montesino Semper

Servicio de Urología. Hospital Virgen del Camino

C/ Irunlarrea, 4 - 31008 Pamplona (Navarra)

e-mail: mmontes@cfnavarra.es

(Trabajo recibido el 18 noviembre 2004) 